\title{
Propelling drug research by physical chemistry
}

\author{
Vesna Gabelica Marković \\ Fidelta d.o.o. Prilaz baruna Filipovića 29, HR-10000 Zagreb, Croatia \\ E-mail: vesna.gabelicamarkovic@glpg.com; Tel.: +385-1-88886 308; Fax: +385-1-8886 444
}

Received: March XX, 2013; Revised: April XX, 2013; Published: April XX, 2013 mmk

\section{Physico Chemical Methods in Drug Discovery and Development. Zoran Mandić (Editor), IAPC Publishing, 2012.}

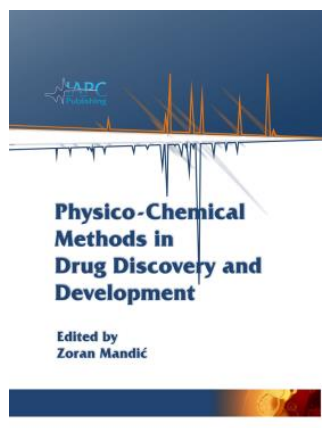

Physico-Chemical Methods in Drug Discovery and Development is a compilation of the key methods and techniques in drug research and development. The book comprises nine chapters written by 15 authors from international academic and industrial laboratories. Compliment to the editor for selecting the authors, who are more than excellent. The chapters cover a wide variety of topics and provide a broad introduction to any individual working on the physicochemical characterisation of compounds in drug research. The first chapter provides a comprehensive review of the theoretical background of physicochemical parameters, as well as methods for their determination. The second chapter of the book describes the application of HPLC as a powerful technique for measuring physicochemical parameters. Basic principles of chromatographic retention and HPLC methodology are used to determine various physico-chemical properties. There is an extension to bio-mimetic chromatography, followed by the application of the data to predict human in vivo distribution. Chapter 3 discusses the importance of and the different approaches to determining permeability. The main focus of this chapter is experimental models and procedures available in early drug discovery. The chapter that follows describes the protein-ligand interaction as an important method used in early drug discovery. There is a summary of all the biophysical methods currently in use, followed by a few brief case studies. Chapter 5 provides a detailed and comprehensive review regarding the application of NMR spectroscopy in the study of ligand-receptor interactions. The main focus is on the NMR techniques applied to studying the bioactive molecules with their biological receptors. Chapter 6 describes chiral separation for enantiomeric determination in the pharmaceutical industry and is focused on the types of molecular interactions. A significant list of selected compounds for the separation of their racemic mixtures is presented, as well as a large reference list. The next chapter presents the importance of choosing the optimal solid form of a drug, an unavoidable topic for this type of book. It includes the thermodynamics of 
different forms, as well as form screening and selection. Thermal analysis and calorimetric methods, which are basic methods currently used in the pharmaceutical industry, are detailed in Chapter 8. This comprehensive chapter includes theory and instrumentations, followed by a few examples of application. The last chapter discusses the importance of near infrared spectroscopy in pharmaceutical research and development. In addition to a theoretical description, near infrared spectroscopy is presented as the technique of the greatest significance for process analytical technology.

The strength of this book lies in its excellent review of numerous physicochemical methods. Overall, the writing style is very easy to follow and the chapters are illustrated where appropriate. It is a very valuable book for anyone interested in physicochemical methods in drug discovery and development. The book is excellent for students and will become an essential reference for any individual working in the pharmaceutical industry.

\footnotetext{
${ }^{\circ} 2013$ by the authors; licensee IAPC, Zagreb, Croatia. This article is an open-access article distributed under the terms and conditions of the Creative Commons Attribution license (http://creativecommons.org/licenses/by/3.0/) (cc)) EY
} 